\title{
A Review: Composition of Neonatal Meconium Microbiota and Its Role for Potential Probiotic
}

\author{
Devi Oktaviyani, Raden Zulfa 'Alawiyyah, Putri Nusaiba, Amarila Malik" \\ Faculty of Pharmacy, Universitas Indonesia, Depok, West Java, Indonesia
}

\begin{abstract}
Early-life period ( $\leq 1$ month after birth) is critical for determining long- and short-term health of neonates. Composition of neonates' gut microbiota varies greatly between individuals whose development is influenced by various factors including differences in maternal diet and lifestyle during pregnancy, related to population and ethnicity. Balanced microbial composition can create symbiosis among commensal microbes, immunomodulatory compound production, and subsequent immune response regulation. Unbalanced microbiota composition, characterized by more pathogenic organism, less diversity, and less resistance to disease, is called dysbiosis. Probiotic bacteria are a bacteria group contributing to the balance of neonates' gut microbiota. Probiotic bacterial strains, such as Lactobacillus, Bifidobacterium and Streptococcus strains, are present in neonatal meconium microbiota. Meconium, a biological material formed during pregnancy, is a useful source of information in describing in utero microbial environment. This review aims to describe probiotic potential in profile composition of neonates' microbiota meconium of multiple ethnicities as marker of neonates' health level. Molecular-based sequencing method, such as Next-Generation Sequencing (NGS), is the preferred method for analyzing complex microbiota communities, such as gut microbiota. Neonatal meconium samples are collected and DNA extractions are carried out, then the target genes are amplified by PCR. The amplicons obtained are sequenced and characterized to determine the presence of potential probiotic strains in sample. Whether the probiotic strains can be used to measure neonates' health level during period of growth and development is also described. Those probiotic strains could be developed as microbial therapeutic agent in gastrointestinal tract disorder therapy.
\end{abstract}

Keywords: diet; meconium; gut microbiota; NGS; probiotic

*corresponding author

Email: amarila.malik@ui.ac.id

\section{INTRODUCTION}

At early-life period the colonization of microbiota in the human gut tract determines the formation of the microbiota in the gut that can affect the development of the body functions in short-term and long-term health. Meconium is a biologic material which is formed during pregnancy, it has been regarded as a source of information that is very helpful to describe the environment of microbes in utero. The first feces of the baby that is issued 24-48 hours after birth is called meconium, which is a representative of neonatal gut environment during the period of pregnancy until their early life period. Several countries have conducted many researches on the meconium microbiota of neonates, but in Indonesia the research on the meconium microbiota is still very rare. Based on the research that is carried out by Malik et al, the profile microbiota of neonates in Indonesia which dominates meconium neonate isolates is Staphylococcus (53.24\%), Bacillus (19.48\%) and Enterococcus faecalis (2.59\%) (Malik, 2020). Microbiota of neonates in India is characterized by the higher abundance of Bifidobacterium and Lactobacillus (Xu et al., 2020). It is demonstrated that the bacteria strains which have the probiotics activity are strains of Lactobacillus, Bifidobacterium, Streptococcus, and Enterococcus in the microbiota of meconium neonates (Zavisic et al., 2019).

A symbiotic state is a condition in which the composition of the gut microbiota is balanced and harmonious and provides benefits for the health of the host (Gupta et al., 2013). The condition when the composition of the microbiota becomes unbalance, which is characterized by pathogenic organism, narrow diversity, less resistant to disease and the lack of ability to combat, is called dysbiosis (Groer et al., 2014). Various studies have reported that dysbiosis in infants is correlated with the state of the pro-inflammatory chronic such as that is seen in allergy, obesity, inflammatory bowel disease (IBD) at the time of adulthood (Fabricatore et al., 2011; Jostins et al., 2012). It's important to create healthy microbiota and symbiosis during the early life of the neonate to support the development of the neonatal health. A group of bacteria that give a contribution to the balance of neonatal gut microbiota in supporting the development of the health of the host is called probiotic bacteria (Sihotang and Fachrial, 2020). 
Human gut microbiota develops soon after birth and can be obtained by variation of interindividual against intrinsic and the environment exposure. There are several factors identified affecting gut microbiota, based on early evidence, which are the states of the host genetic, and early exposure including mode of delivery, antibiotics, and diet that affect neonates gut microbiota (Stearns et al., 2017). However, variations in inter-individual and multi-ethnic with different patterns of diet are not yet evaluated comprehensively. Therefore, the aim of this review article is to show the composition of the microbiota of meconium neonates, especially probiotic bacteria potential that is contained inside of a difference diet and life style of the mother during pregnancy, is related to population and ethnicity with analysis using technology Next Generation Sequencing (NGS). The sequencing-based molecular method, such as NGS, is the method of choice for analyzing complex community of microbiota (Bleich and Fox, 2015). Availability of NGS allows observation of genes and genomes contained in the complex microbiota community including gut microbiota. The use of the technology of molecular and bioinformatics analysis is considered to give a contribution to the development of the neonatal gut microbiota.

At the end of the writing, the readers are expected to know probiotic potential in the profile composition of microbiota meconium neonates of multiple ethnicities that can be used as a marker level of neonatal health. Thus, probiotic bacteria potential in the microbiota of meconium neonates is capable to become the starting measure for assessment of potential health of newborns in supporting the health of the newborn during the critical growth and development. In addition, the probiotic bacteria potential can be developed as a therapeutic microbial agent for digestion tract diseases.

\section{METHODS}

Literature search was performed using the keyword "(Probiotic) AND (Gut microbiota) AND (Meconium)" on the Google Scholar and Science Direct databases. To be included in this review article, studies had to be published within these recent 10 years. Inclusion criteria used to refine searches among others were: the study is an observational study that identifies the composition of the neonatal gut microbiota which are associated with the development of the neonatal health, and the study of probiotics bacteria potential in the gut. While the exclusion criteria used were articles that are not able to be accessed and articles in languages other than Indonesian and English.

Searching was carried out using Google Scholar and Science Direct data bases. In addition, screening article was carried out to search for articles that are relevant and not a duplication in various data bases. After finding a number of articles that correspond to the inclusion and exclusion criteria, 15 articles are analyzed and incorporated into this review. The literature search process can be seen in Figure 1. The method of profiling potential probiotics in the neonatal meconium microbiota is by grouping several strains of bacteria found from previous studies of a particular ethnicity in a country then the data is presented in tabular form.

\section{Probiotic Bacteria}

Probiotic bacteria are defined as living organisms which, if granted in adequate amount, provide benefits the health of the host (Boyle, Robins-browne and Tang, 2018). Bacterial probiotics in the gut can affect the intestine mucous through several ways, namely by increasing the production of mucin, affecting the absorption of fluids, strengthening the structure of the mucosa, and affecting apoptosis and the immune system. Each species of lactic acid bacteria (LAB) has the distinct effect of probiotics (Sihotang and Fachrial, 2020). There are several factors that affect the proportion of neonatal gut probiotics, such as mode of delivery, type of nutrition, gestational ages, inpatient hospitalization, and the use of antibiotics by neonates (Rodríguez et al., 2015).

Probiotic bacteria such as Bifidobacterium longum and $B$. infantis or Lactobacillus acidophilus have been routinely used as prophylaxis in several NICUs in Europe such as Austria, Netherlands, and Germany. Bacterial probiotics are expected to reduce gut dysbiosis and the rate of infection, and to increase metabolism. Probiotics also do not cause adverse side reaction which is not desirable. Probiotics are believed to reduce the risk of health problem in the short-term, but the evidence and the convincing clinical trial are not yet available (Marißen et al., 2019).

In order to identify the presence of probiotic bacteria potential in profile of the microbiota meconium of neonates, evaluation of the bacteria obtained from meconium can be done. The natural probiotics can be identified using traditional methods of phenotype and genotype, which include identification of morphology and physiology of probiotics, in vitro and in vivo test, the resilience of life in conditions of artificial gastrointestinal, adhesion to Hexadecane test, and the antimicrobial activity test (Zavisic et al., 2019). Primary criteria in the selection and application of probiotics is the resilience of bacterial cells through digestive tract, the ability to stick (adhesion) on the epithelial cells of host digestive tract and the ability to colonize in different parts of the gastrointestinal tract (Kaushik et al., 2009; Fijan, 2016). 


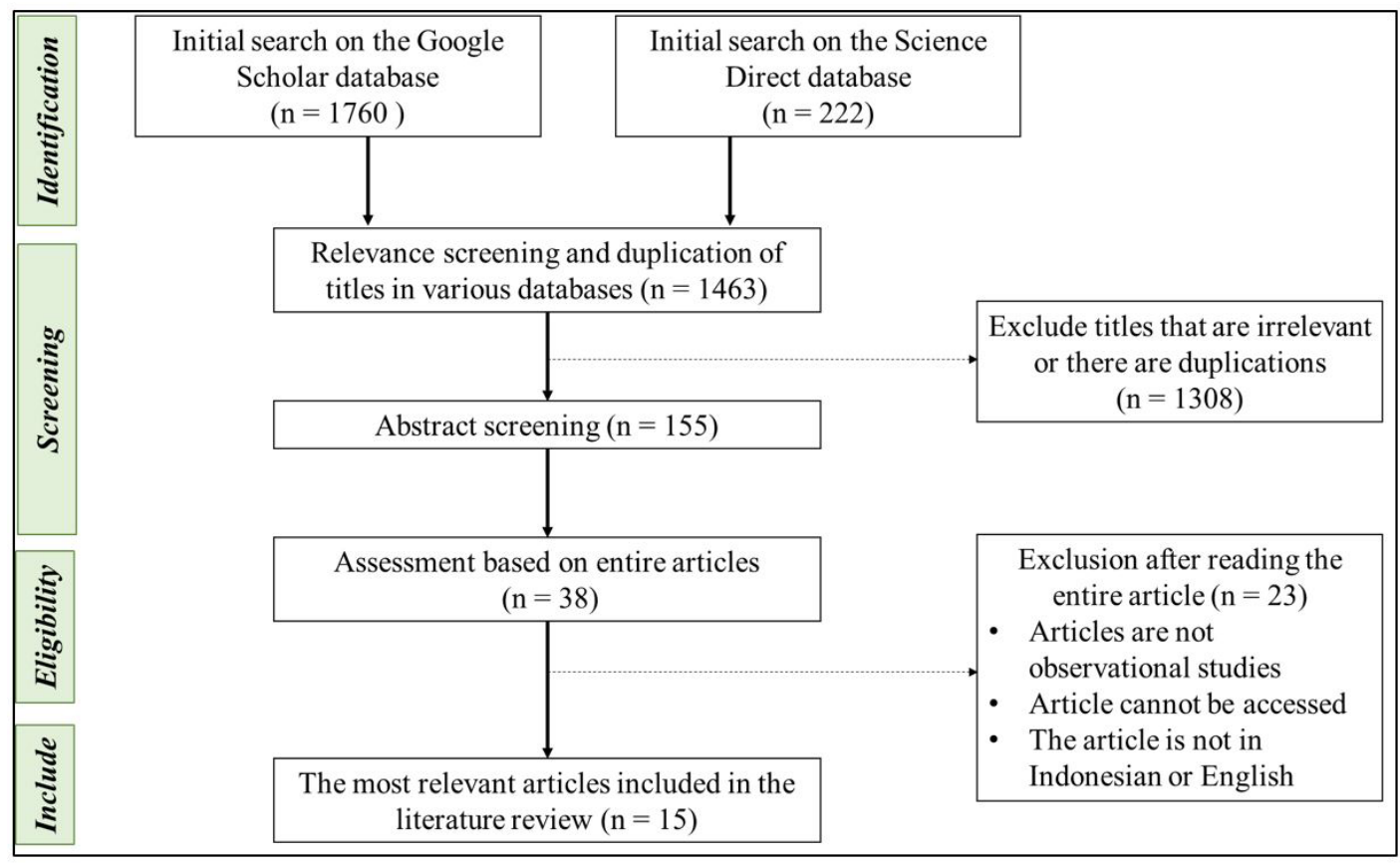

Figure 1. Literature search flow

Important characteristics from the probiotic potential also involve auto-aggregation, hydrophobicity of the cell surface, no toxicity and bacteria translocation (Campos et al., 2006; Fakruddin, Hossain and Ahmed, 2017). In addition, the activity of antimicrobials against different pathogens is another important aspect of probiotics, which are associated with changes in gut microbiota and has been used to suppress the growth of pathogens. It is because probiotics can produce antimicrobial compounds (organic acid, hydrogen peroxide and bacteriocin) and the competition to get the nutrients with pathogens on human's gut (Shokryazdan et al., 2014; Boyle, Robinsbrowne and Tang, 2018).

\section{Composition of Neonatal Meconium Microbiota}

Microbiota is an entire population or information genetic of all the microorganisms that settle on a specific environment (Bleich and Fox, 2015). Microbiota composition can be composed of many bacteria that are significantly different, about 160 bacteria species per sample feces, and this ecosystem has important role in the health of human being. Changes in the composition of the gut microbiota in neonates have been shown in several studies to be associated with the occurrence of disease later in life (Rodríguez et al., 2015).

Both meconium and feces can be used as non-invasive methods for analyzing the gut microbiota. Meconium is a first feces of the baby that is issued 24-48 hours after birth and considered as representative of intrauterine environmental, and feces that is issued a few days after birth can be considered to reflect the exposure on the outside of the uterus (Mueller et al., 2017).
In general, at the age one week of the first life, the environmental microbiota of the intestine is widely colonized by Actinobacteria (including Bifidobacterium), Proteobacteria, Bacteroides, and slightly colonized by Firmicutes (including Lactobacillus spp; that dominate the flora vaginal). In contrast, neonates that are born with body weight $<1200 \mathrm{~g}$ is primarily colonized by Firmicutes and Tenericutes, and slightly colonized by Actinobacteria. Bacteria in neonatal meconium, such as Escherichia coli, Enterococcus faecium, and Staphylococcus epidermidis, can be found due to translocation of gut microbiota through the blood (Gonza, 2019).

The diversity and composition of the microbiota in neonates are related to several factors, both maternal and neonatal factors. Maternal factors include maternal diet during pregnancy, mother's body weight, and antibiotics intrapartum. Meanwhile, neonatal factors include mode of delivery, gestational age, birth weight, breastfeeding patterns, and use of antibiotics (Matamoros et al., 2013). Healthy gut microbiota (symbiosis) of neonates are microbiota that are derived from neonatal terms, born through the vagina, free of antibiotics and breast milk. Dysbiosis is a deviation from the healthy state by decreasing the diversity of microbes and the lack of capacity to control pathogenic and drug-resistant organisms (Marißen et al., 2019).

Apart from these factors, the neonatal gut microbiota is also influenced by ethnicity. Ethnicity refers to the group of people who have a commonality of race, culture, 
religion, or similar other characteristics. Ethnicity provides several biological and cultural factors and can be characterized using a number of difference parameter (dietary habits, country of origin, etc.). Dietary habit affects the difference metabolic strategy, which subsequently affects the microbiota of neonates coming from different ethnic groups (Stearns et al., 2017).

\section{Molecular Analysis Methods of Neonatal Meconium Microbiota}

\section{Identification method}

There are several methods for the identification of gut microbiota that can be used. ranging from conventionally culture-based methods to molecular-based methods by using nucleic acid, which has grown with leaps and bounds in the past several decades. Each method has their disadvantages and advantages. Culture-based methods have advantages, namely simple, semi-quantitative, and more economical, but most of gut bacteria is an anaerobic bacteria that is difficult to be cultured. Therefore, the molecular-based method using nucleic acid (DNA/RNA) appears. The molecular-based analysis method consists of non-sequencing methods (FISH/Fluorescence in situ hybridization flow cytometry; DGGE/Denaturing gradient gel electrophoresis; DGGE/Temperature gradient gel electrophoresis) and sequencing (ge16S rRNA or shotgun microbiome). The advantages of this molecular method are more rapid, quantitative, and identification of bacteria to the level of the taxonomy that is difficult to achieve with the culture-based methods. However, the use of molecular-based methods requires high power of computing and analysis (Sarangi, Goel, \& Aggarwal, 2019).

Researches about the microbiota recently have most often characterized the presence of bacteria in the samples with sequencing fragments of 16S rRNA genes coding. Genes coding for rRNA is the gene most sustainable (conserved) so that the sequences of genes are often chosen for the study of bacterial taxonomy and phylogeny because it can give a high resolution of taxonomy (Goodrich et al., 2014). Genes $16 \mathrm{~S}$ rRNA contains nine regions that are hypervariable (V1 - V9) ranging from about 30 to 100 pairs of bases that are involved in the structure of the secondary of the small subunit ribosomal. In the hypervariable area, interspersed regions are sustainable. The regions that are more sustainable are correlated with the high level of taxonomy, and regions that are less sustainable have taxonomy level that is much lower such as genus and species. In doing taxonomy, classification is quite simply done by sequencing each hypervariable region, while regions that are sustainable allow for the design of universal primers (Caporaso et al., 2010; Youssef et al., 2009).
Sequencing of the 16S rRNA gene has the disadvantage that the determination of bacteria which exist in a sample is limited to analysis sequence information of taxonomic, which is included in the database of reference of $16 \mathrm{~S}$ rRNA, but not able to directly assess the biological function from the community of microbes in a sample (Sarangi, Goel and Aggarwal, 2019). Besides that, sequencing of $16 \mathrm{~S}$ rRNA gene is generally not able to distinguish between cells of the bacteria that live and metabolic active, damaged or dead, or DNAfree. It is important for the innate immune system of human to be able to selectively respond to the viability of microbes (Diggikar, 2019). The alternative is with meta-genomic and meta-transcriptomic shotgun. Metagenomics is defined as the application of modern genetics to identify microbes to the level of species in their natural environment naturally without the need of culturing (Diggikar, 2019). Meta-genomic and metatranscriptomic shotguns can provide a functional profile of the microbiome and the resulting taxonomic resolution is down to the line level (Luo et al., 2015; Cleary et al., 2016). This makes it possible to obtain information about the transcription activity of the microbiota community (Franzosa et al., 2014).

\section{Next-Generation Sequencing (NGS)}

Next-Generation Sequencing (NGS) is a technology that is potentially able to replace a lot of the workflow of conventional microbiology and now this method is the method of choice to analyze the microbiota composition of gut (Besser et al., 2018; Rintala et al., 2017). This technique mainly depends on using the 16S rRNA gene. First-Generation Sequencing (1977), also known as "Sanger" Sequencing is mainly based on DNA denaturation by gel electrophoresis, which is time consuming and expensive. It led to the emergence of NGS or Second-Generation Sequencing (SGS) in the year 2005 and has revolutionized the identification of the microbiome (Goodwin, Mcpherson and Mccombie, 2016). Basic characteristics of SGS technology is short processing millions of reading in parallel, speeding up the process of sequencing compared to the first generation, the low cost of sequencing and sequencing output, and can be detected directly without requiring electrophoresis, for example genome sequencer (GS) FLX + titanium, and GS junior systems, genome IIx, Illumina: HiSeq and MiSeq (Sarangi, Goel and Aggarwal, 2019).

Bioinformatics Profiling of Neonates Gut Microbiota Samples of community, for example meconium or stool that describe the gut environment, are collected and DNA is extracted from each sample. Genomic markers, that are informative in taxonomy and common to almost all organisms, are then targeted and treated with amplification by PCR (Polymerase Chain Reaction). In 
Table 1. Strain of probiotic potential on gut microbiota meconium

\begin{tabular}{|c|c|c|c|c|c|}
\hline $\begin{array}{l}\text { Ethnicity } \\
\text { (Country) }\end{array}$ & Sample & Molecular Identification & $\begin{array}{l}\text { Potential } \\
\text { Probiotic } \\
\text { Strains }\end{array}$ & $\begin{array}{l}\text { Probiotic } \\
\text { Potential } \\
\text { Test }\end{array}$ & Ref \\
\hline $\begin{array}{l}\text { Belgrade, } \\
\text { Serbia }\end{array}$ & $\begin{array}{l}\text { Meconium } \\
(\mathrm{n}=10)\end{array}$ & $\begin{array}{ll}\text { - } & \text { Isolation of lactic acid } \\
\text { bacterial (culture method) } \\
\text { - } & \text { DNA Genomic extraction } \\
\text { - } & \text { Amplification of the 16S rDNA } \\
\text { - } & \text { gene by PCR } \\
\text { - } & \text { Sequencing using the di- } \\
\text { deoxynucleotide DNA chain } \\
\text { termination method }\end{array}$ & $\begin{array}{l}\text { Lactobacillis } \\
\text { fermentum } \mathrm{G}-4\end{array}$ & $\begin{array}{l}\text { It's } \\
\text { been tested }\end{array}$ & $\begin{array}{l}\text { (Zavisic et } \\
\text { al., 2019) }\end{array}$ \\
\hline France & $\begin{array}{l}\text { Meconium } \\
(\mathrm{n}=6)\end{array}$ & $\begin{array}{ll}\text { - } & \text { Isolation of lactic acid } \\
\text { - } & \text { bacterial (culture method) } \\
\text { - } & \text { Amplification of the 16S rDNA } \\
\text { gene by PCR } \\
\text { - } & \begin{array}{l}1 \% \text { agarose gel electrophoresis } \\
\text { - }\end{array} \\
\text { Sequencing }\end{array}$ & E. faecalis & $\begin{array}{l}\text { It's } \\
\text { been tested }\end{array}$ & $\begin{array}{l}\text { (Atya et al., } \\
2015)\end{array}$ \\
\hline China & $\begin{array}{l}\text { Meconium } \\
(\mathrm{n}=18)\end{array}$ & $\begin{array}{ll}\text { - } & \text { Extraction of genomic DNA samples } \\
\text { - } & \text { Metagenomic sequencing (Illumina } \\
\text { Hiseq2500) }\end{array}$ & $\begin{array}{l}\text { Bacillus and } \\
\text { Lactobacillus } \\
\text { (Firmicutes } 37.4 \% \text { ) }\end{array}$ & $\begin{array}{l}\text { Not tested } \\
\text { yet }\end{array}$ & $\begin{array}{l}\text { (Birarra, } \\
\text { Heye and } \\
\text { Shibeshi, } \\
\text { 2017) }\end{array}$ \\
\hline $\begin{array}{l}\text { Tokyo, } \\
\text { Japan }\end{array}$ & $\begin{array}{l}\text { Meconium } \\
(\mathrm{n}=151)\end{array}$ & $\begin{array}{ll}\text { - } & \text { RNA extraction } \\
\text { - } & \text { Reverse transcription-quantitative- } \\
& \text { PCR (RT-qPCR) }\end{array}$ & $\begin{array}{l}\text { Bifidobacterium } \\
\text { and Lactobacillus } \\
(30-35 \%)\end{array}$ & $\begin{array}{l}\text { Not tested } \\
\text { yet }\end{array}$ & $\begin{array}{l}\text { (Nagpal et } \\
\text { al., 2016) }\end{array}$ \\
\hline $\begin{array}{l}\text { Lisbon, } \\
\text { Portugal }\end{array}$ & $\begin{array}{l}\text { Meconium } \\
(\mathrm{n}=117)\end{array}$ & $\begin{array}{ll}- & \text { Extraction of genomic DNA samples } \\
- & \text { Reverse transcription- } \\
\text { - } & \text { quantitative -PCR (RT-qPCR) } \\
\text { 16S rRNA gene sequencing }\end{array}$ & Lactobacillus & $\begin{array}{l}\text { Not tested } \\
\text { yet }\end{array}$ & $\begin{array}{l}\text { (Morais et } \\
\text { al., 2020) }\end{array}$ \\
\hline Indonesia & $\begin{array}{l}\text { Meconium } \\
(\mathrm{n}=14)\end{array}$ & 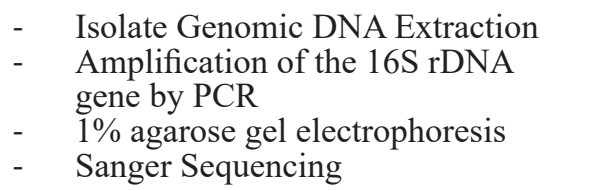 & $\begin{array}{l}\text { Bacillus } \\
\text { subtilis strain MBF } \\
30 \text { and Enterococcus } \\
\text { faecalis strain MBF } 66 \\
\text { and MBF } 67\end{array}$ & $\begin{array}{l}\text { Not tested } \\
\text { yet }\end{array}$ & $\begin{array}{l}\text { (Malik, } \\
\text { 2020) }\end{array}$ \\
\hline $\begin{array}{l}\text { Valensia, } \\
\text { Spain }\end{array}$ & $\begin{array}{l}\text { Meconium } \\
(\mathrm{n}=20)\end{array}$ & 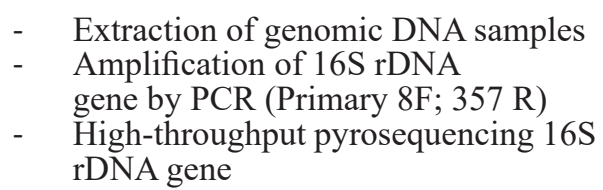 & $\begin{array}{l}\text { Enterococcus }(16.79 \%) \\
\text { Lactococcus }(14.01 \%) \\
\text { Streptococcus } \\
(6.34 \%)\end{array}$ & $\begin{array}{l}\text { Not tested } \\
\text { yet }\end{array}$ & $\begin{array}{l}\text { (Gosalbes } \\
\text { et al., } \\
2013 \text { ) }\end{array}$ \\
\hline
\end{tabular}

microbial research, amplicon sequencing typically targets the $16 \mathrm{~S}$ ribosomal RNA (16S rRNA) gene subunit, which is a taxonomically and phylogenetically informative marker. Amplicons are produced in the sequencing and then their bioinformatics are characterized to determine which microbes exist in the sample and how their relative abundances are (Collado et al., 2016; Hugenholtz \& Pace, 1996; Marshall, 1977).

The raw data of sequence, which are obtained from the sequencing, contains sequence adapters and primers used in amplification process. By thus, the first step in the data analysis is to cut the sequences from those adapters and primers. If using a paired-end sequencing technique, where DNA is sequenced in two directions (forward and reverse), the next step is to combine the pairs of readings into one. It is done to get the reading results with more length and help eliminate readings with low quality, and then to obtain good quality sequences. The diversity of bacteria species in any sample is clustered by 97\% similarity sequence of DNA into OTU (Operational Taxonomic Units) (Sarangi, Goel and Aggarwal, 2019).

Statistical analysis of the $16 \mathrm{~S}$ rRNA gene sequence data is carried out together with statistical tools (QIIME and JMP Pro 12) (Rintala et al., 2017). All analysis are conducted from the OTU table which are randomly inserted. Bacterial diversity of the sample can be seen by analyzing alpha diversity ( $\alpha$-diversity) for the diversity of bacterial species in one sample and analysis of beta 


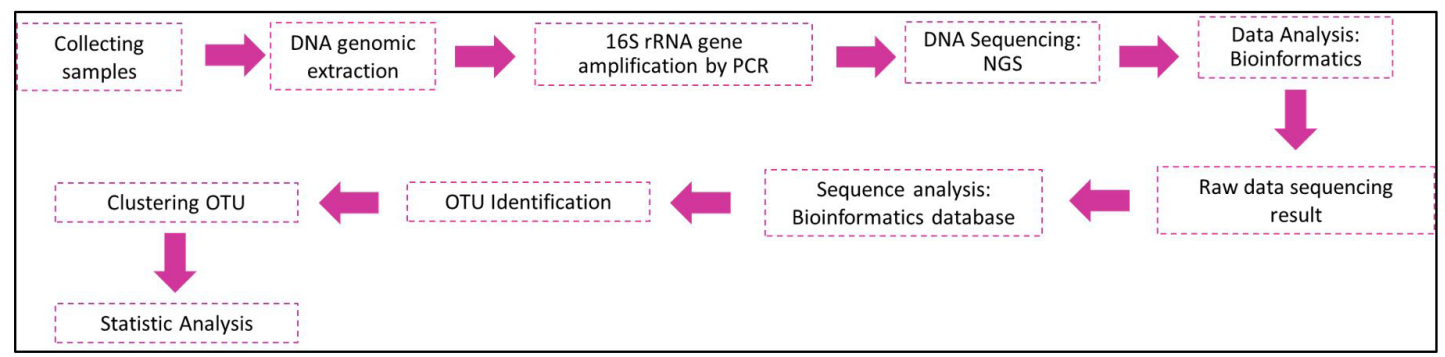

Figure 2. Workflow for analysis of neonatal meconium microbiota based on molecular Next Generation Sequencing (NGS) technology

diversity ( $\beta$-diversity) which represents an explicit comparison of microbial communities based on their composition or diversity of bacterial species between samples (Alcon-giner et al., 2017). Differences in taxonomy richness in an abundance of OTU groups which are significant in statistics can be done with the Kruskal-Wallis test, the average index of Shannon, Steel-test Dwass All Pairs, and other statistical tests. Differences of diversity of all bacteria in the entire sample are analyzed by using matrix distance weighted UniFrac generated from OTU table, and visualized with the principal coordinate analysis $(\mathrm{PCoA})$ (Rintala et al., 2017). Figure 1. shows the workflow of neonatal meconium microbiota analysis based on the sequencing of 16S rRNA gene using Next Generation Sequencing (NGS) molecular technology, which is most widely used in microbiota community analysis.

\section{Phylogenetic Identification of Neonatal Meconium Probiotic Bacteria}

Phylogenetic analysis can be carried out at various levels and combined with other characteristics (phenotypes) as needed to make a definitive taxonomic classification (O’Sullivan, 1999). Most Lactic Acid Bacteria (LAB) have the probiotic activity. Phylogenetically they are from the phylum of Firmicutes, class of Bacilli, order of Lactobacillales, and family of Lactobacillaeceae (Heilig et al., 2002). The taxonomy of LAB is quite complicated, so to find out the genetic relationship or linkages between these bacteria can be done by creating a phylogenetic tree based on the closest sequence that has been identified in the sequence database (GenBank) by BLAST (Basic Local Alignment Search Tool). Sequences of the 16S rRNA gene were obtained from the results of sequencing the isolates of potential probiotic bacteria compared with sequences in the database of NCBI BLAST, where identification is based on homology to sequences of $16 \mathrm{~S}$ rRNA (Heilig et al., 2002).

Based on the research that is carried out by Kook et al., tree phylogenetic which shows the genetic relationship between isolates of potential probiotic bacteria of meconium is obtained, such as that shown in Figure 2. Phylogenetically, isolates of gut probiotic bacteria show that the percentage of Lactobacillus is relatively high (Kook et al., 2019). Lactobacilli and Bifidobacteria are two groups of important probiotics, because they are proven to be a member of the commensal human microflora, history of safe use and general evidence supporting their positive role. Phylogenetically, it has been observed that 18 species of Lactobacilli and 31 species of Bifidobacterium, 11 of whom have been detected in the human feces, are probiotics (Tannock, 1999).

\section{RESULTS AND DISCUSSION}

The Role of Probiotics in the Meconium Microbiota and Its Implication to the Health of Neonates

Several studies recently have reported that probiotics are beneficial to the host by improving the balance of gut microbiota and immunity of host directly or indirectly (Muñoz-Atienza et al., 2013; Varankovich et al., 2015). Beneficial effects of probiotics depend on strains, then different strain from the same species of probiotics can give the different effect (Butel, 2014). Therefore, it is important to understand how probiotics modulate interactions with host immunity by a mutualistic way to provide the balance of environment rich in nutrients for gut microbiota that can increase the efficiency of digestion and function of immune hosts (Lee and Mazmanian, 2010; Hooper et al., 2012).

a) Microbiota and Immunity of Neonates

One hypothesis suggests that the maternal gut microbiota may be translocated to the fetus via the bloodstream. Neonate after birth is immediately exposed and colonized quickly by the microbiota of environments that vary, such as from the vagina, skin, and the environment during the process of birth, the time in which the activity of Toll like receptor (TLR) is reduced to facilitate the colonization process (Clemente et al., 2012). Microbiota helps maintain the normal immune function of the host through the expression of MAMP (Microbe-Associated Molecular Patterns), metabolites, and antigens (Belkaid and Hand, 2014). The mother's microbiota and its products can transfer to the fetus and also provide optimal host and microbial mutualism interactions at birth, not 
only increasing the antibacterial immune response (de Aguero et al., 2016). Therefore, the host immune system is very dependent on the commensal bacteria to protect against pathogens invasion.

Innate immune system consists of several types of cells such as dendritic cells, macrophage, innate lymphocytes cells and epithelial cells, which first interacted with gut microbiota and form a first line system to attack the pathogen. Dendritic cells can recognize microbial factors through activation of innate immune receptors which further polarize the adaptive immune response (Smolinska et al., 2017). Helicobacter pylori infection can interfere the normal biosynthesis of retinoic acid by dendritic cell which led to the development of chronic gastritis (Bimezok et al., 2015). Macrophages are myeloid phagocytes that are responsible for removing host cell debris, foreign substances, and working in the presence of microbial colonization (Bain et al., 2014). Innate lymphocyte cells (ILC) are a branch of the innate immune system, and their function can be determined by commensal microbial colonization. Commensal bacteria can induce the expression of IL-22 by ILC3, which subsequently induces the expression of fucosyltransferase 2 to fucosylation of the protein surface that is crucial for the defense of the host against the enteric pathogens in mice (Goto et al., 2014). Intestinal epithelial cells form a mucosal barrier that protects the host from pathogen invasion and toxic agents (Smolinska et al., 2017). Specific goblet epithelial cells produce mucins that work as a component structure of the primary layer of mucus that protects the epithelial cells against pathogens. Other epithelial cells such as Paneth cells are the producer of antimicrobial peptides such as defensins and lysozymes (Gassler, 2017).

Innate immunity system is still less specific to the antigen than adaptive immune system so it is still not known how the specific genus/species of microbes or molecular effectors of microbial trigger the innate immune system. By thus, it is important to understand the interaction between specific species of microbe and innate immunity function to prevent the development of a disease. The adaptive immune system is activated when it receives polarizing signals from innate immune cells to induce specific lymphocyte responses to bacteria and metabolic factors (Zhao and Elson, 2018). Response immune adaptive to microbes consists of two types: immune cell-mediated by cell $\mathrm{T}$, and immunity humoral mediated by cell B that produces antibodies (Russo, et al., 2016).

Cells $\mathrm{T}$ naïve is a critical component of the adaptive immune system, which can differentiate either into cell $\mathrm{T}$ effector that fight pathogens (Weaver et al., 2006), or into cell regulatory $\mathrm{T}$ (Treg), which tolerate bacteria in the gut and prevent autoimmunity (Barnes and Pownie, 2009). T helper cells (Th17) is an effector T cell CD4+, which is characterized by the production of IF-17, a cytokine that protects the mucous cells from infection (Hooper, 2012). In contrast to Treg cells, Th17 cells were also found to be a major stimulus in the pathogenesis of autoimmune diseases (Harrington et al., 2005). In mice, intestinal Th17 cells can be induced and accumulated in response to colonization of commensal microbes such as segmented filamentous bacteria (SFB) and E. coli (Atarashi et al., 2015). The gut microbiota acts as either an inflammatory factor or an anti-inflammatory factor. They act as an inflammatory factors by inducing the production of IL$1 \beta$ and IL- 6 by dendritic cells and macrophages which direct the differentiation of Th17 cells (Shaw et al., 2012), while they act as an anti-inflammatory factor by inducing an IL-10 response in intestinal $\mathrm{T}$ cells that prevents the activity of exaggeration of cell Th17 and the potential damage of barrier mucosa in rats (Round et al., 2011). Besides that, the imbalance of Treg cells can cause immune-mediated disease (Bennett et al., 2001), whereas the excessive expression of cells can cause chronic infection or tumorigenesis (Gratz and Campbell, 2014).

$\mathrm{B}$ cells regulate the immunity response mainly by producing IL-10 and differentiation of B cells regulator producing IL-10 that is driven by the microbiota in mice (Rosser et al., 2014). B cells also protects the host against invasion of microbes through the production of antibodies, including IgA secretory that serves as a form of the biggest antibody that exist on the surface of the gut mucous and plays an important role in maintaining gut homeostasis (Mantis et al., 2011). Lack of microbial stimulation, leading to a reduced number of plasma $\operatorname{IgA}+$ cells in the gut and a lack of abundance of IgA (Lécuyer et al., 2014). Research recently have demonstrated that the abundance of bacteria binding $\operatorname{IgA}$, such as E. coli and other Enterobacteriaceae bacteria, in human is related with enteropathy (You et al., 2015) or Crohn's disease (Viladomiu et al., 2017). It is demonstrated that commensal bacteria can interact directly with IgA which leads to the compromise on host health. The research that is carried out by Okai et al, shows that some clones of $\operatorname{IgA}$, which is isolated from the plasma cell of healthy individuals, is selectively bind to the specific pathogen with high affinity, but not to beneficial bacterial (Okai et al., 2016). But it is not yet clear, what type of IgA clones is selectively bind to certain microbes and the factor that determines the affinity between IgA and that microbes (Ma, Suzuki and Guan, 2018).

Most major inventions which have been reported are originated from studies in model experimental animals such as rats or mice. Further research on the gut microbiota and human host, especially neonates is needed 


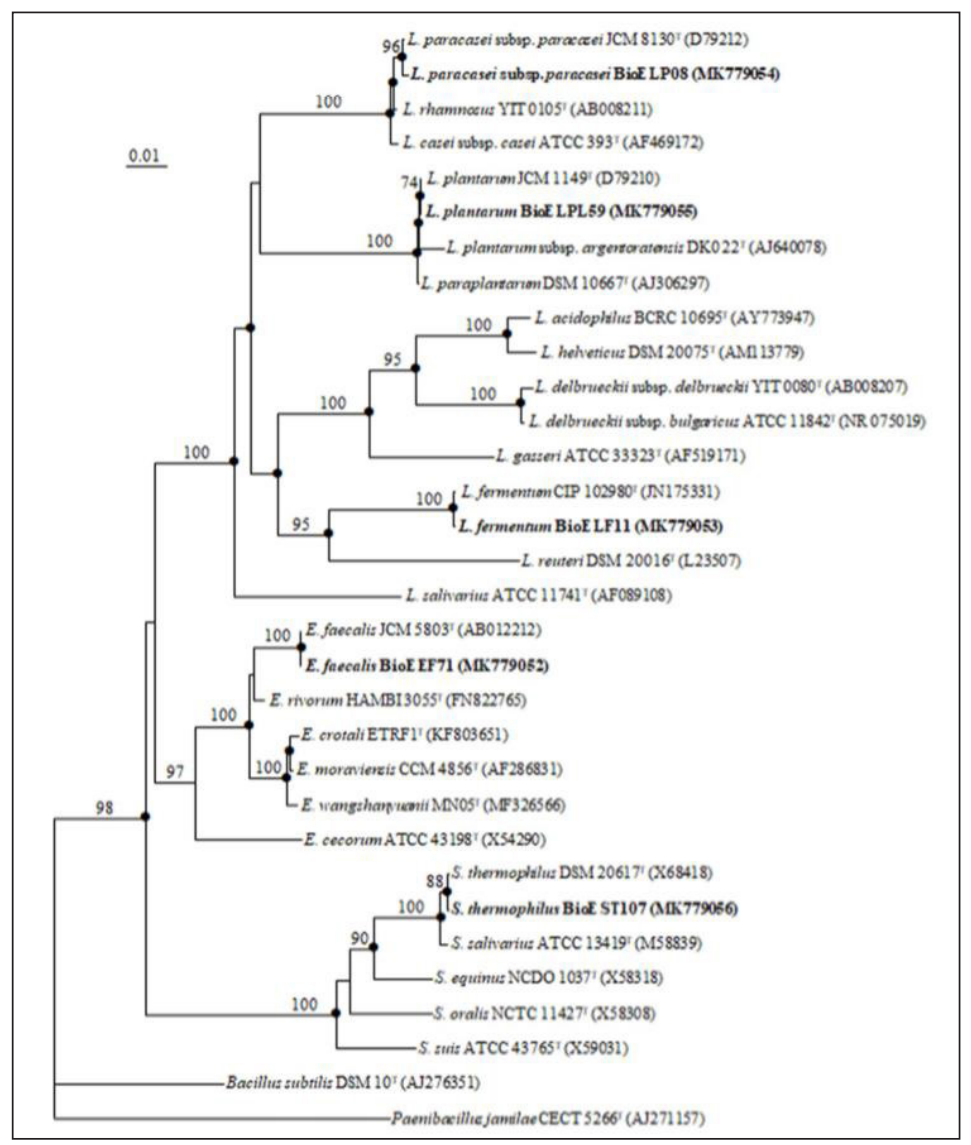

Figure 3. Phylogenetic Tree of Gut Microbiota Probiotic Bacteria (Source Image: Kook et al., 2019)

to describe the role of the gut microbiota in maintaining the normal function of host immunity. Therefore, the results can provide information that is more accurate about how the interaction of gut microbiota with the host immune system is in supporting the health development of host.

b) Mechanism of Action of Probiotics in Supporting Neonate Health

Bacterial probiotics such as Lactobacillus, Bacteroides, and Bifidobacterium can induce Treg cell as described in studies on animals and humans (Dwivedi et al., 2016). Bifidobacterium infantis administration promotes the generation and function of Treg cells, which limits the pro-inflammatory response to Salmonella infection in mice (O'Mahony et al., 2008). The consumption of Bifidobacterium infantis in 35.624 healthy humans resulted in an increase in the proportion of Foxp3 Treg cell expression and increased IL-10 secretion in peripheral blood (Konieczna et al., 2012). Oral administration of Lactobacillus gasseri SBT2055 was reported to induce $\operatorname{IgA}$ production and increase the number of IgA cells in Peyer patches in lamina propria from mice (Sakai, et al., 2014).
The mechanism action of probiotics that can support the health of the host can be divided into three primary categories, namely the interaction directly with the host, inhibiting the growth of pathogens, and modulation response of the immune host such as that shown in Figure 2.

1. Direct interaction with host

Probiotics can directly interact with the intestinal epithelium and dendritic cell from the host, to modulate a signal that leads to the production of mucus and defensins, improvement of tight-junction and the barrier function, and prevention of cytokine induced apoptosis (Schlee et al., 2007; 2008; Yan et al., 2007). Intestinal epithelial cells and dendritic cell interact with microorganisms through PPR (Pattern Recognition Receptors). Macromolecules of bacterial cell wall, such as peptidoglycan in Gram-positive bacteria and lipopolysaccharides in Gram-negative bacteria, are key probiotic ligands that interact with PPR and induce signaling pathways, resulting in probiotic-host crosstalk (Labeer et al., 2010). Pili on the bacterial surface plays an important role in bacterial adhesion to the PPR of the host (Proft and Baker, 2008). Several probiotics, including Lactobacillus, Streptococcus, and Bifidobacterium can 


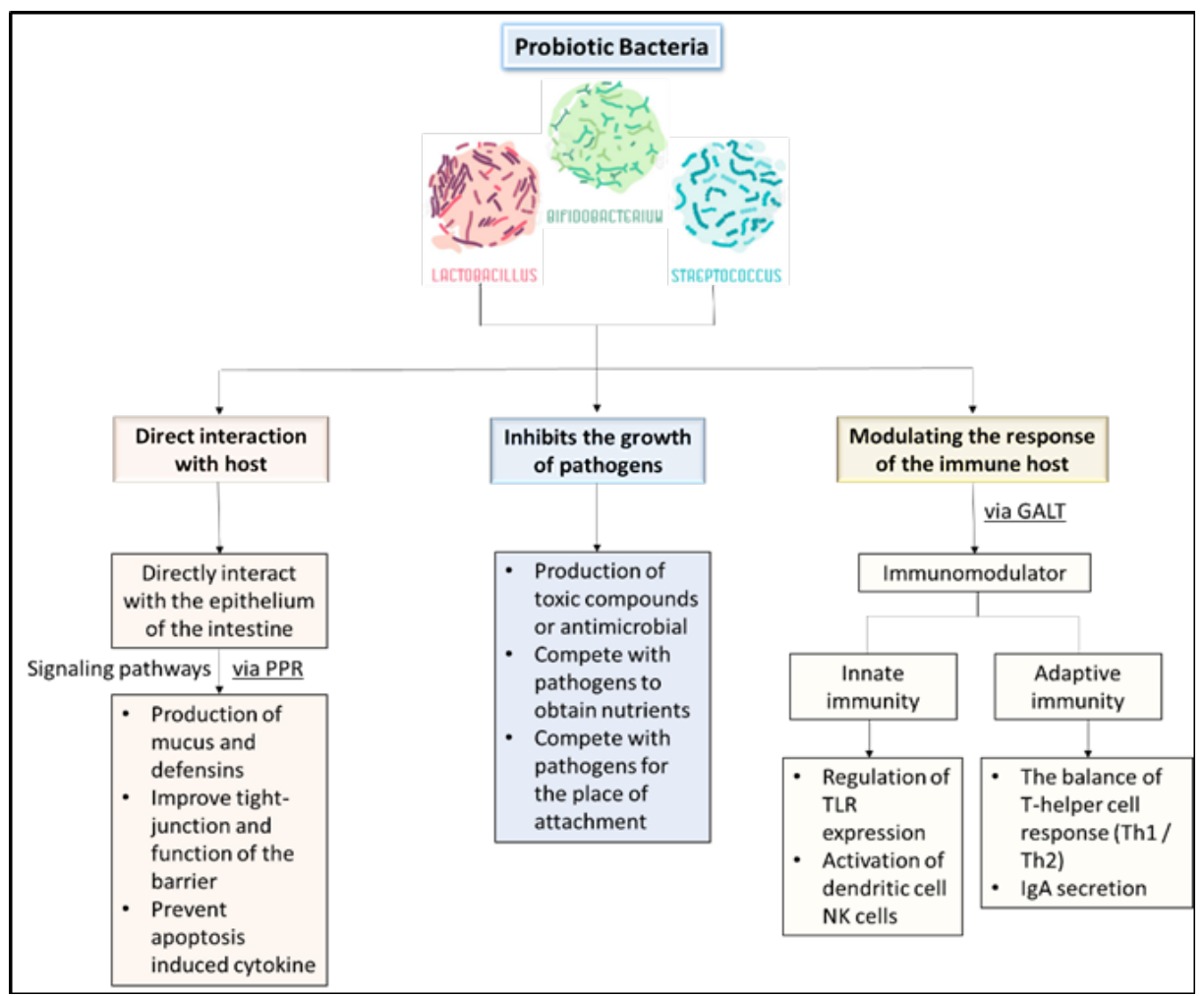

Figure 4. Mechanism of action of probiotics in promoting neonatal health

attach to the epithelial cells of the host intestine and provide probiotic effects (Kankainen et al., 2009, Turroni et al., 2014; Brittan et al., 2015).

2. Inhibition of the pathogen growth

Probiotics can inhibit the growth of pathogens through the production of toxic compounds or antimicrobial, competition with pathogens to obtain nutrients, and attachment to the surface of the intestinal epithelial bacteria and competition with pathogens for the place of attachment.

The production of toxic compounds or antimicrobial, including bacteriocin, acid organic, and hydrogen peroxide, can directly inhibit the growth of pathogens. Lactobacillus salivarius inhibits the growth of Helicobacter pylori and reduces the inflammation response that are induced by Helicobacter pylori in mice (Aiba et al., 1998). Lactobacillus crispatus F117 and Lactobacillus paracasei F2 and F28 inhibit the growth of Staphylococcus aureus through the high production of hydrogen peroxide (Ocana et al., 1999).

Competition with pathogens results to the limitation of nutrition and energy, thereby inhibiting growth and proliferation in the gut (Bajaj et al., 2015). Bifidobacterium adolescentis S2-1 competes with Porphyromonas gingivalis for vitamin $\mathrm{K}$ as a growth factor, thereby inhibiting the growth of periodontal potential pathogens (Hojo et al., 2007).
Probiotics attaches to the surface of the intestinal epithelial cells and competes with pathogens for adhesion sites (Ohland and MacNaughton, 2010). Bacterial adhesins, such as mucus binding protein (MUB), are expressed on the surface of the probiotic to facilitate their interaction with host dendritic cells, thereby increasing their phagocytic capacity (Bene et al., 2017). Some strains/species of Lactobacillus, which can compete with pathogens such as E. coli K88 and Salmonella typhimurium, have been reported to induce mucin MUC3, which reduces the adhesion of E. coli, to lower the $\mathrm{pH}$ tract digestion with the production of acetic acid or lactic acid, and to inhibit the growth of certain pathogens such as Salmonella and E. coli (Mack et al., 1999; Bermudez-Brito et al., 2012).

3. Modulation of the host immune response

Immuno-modulator activities of lactic acid bacteria (LAB) mainly related to the GALT (gut-associated lymphoid tissues). LAB immunomodulatory activity is not only on innate immunity system (regulation of Tolllike receptors expression, activation of dendritic cells, and natural killer cells), but also in adaptive immunity systems including the balance of T-helper cell response (Th1 / Th2) and also IgA secretion (Tsai et al., 2012).

Potential Probiotic Composition of Neonatal Meconium in Several Populations and Ethnicities Researches that characterize the composition of the neonatal gut microbiota from meconium or feces through 
analysis using molecular methods have been growing by leaps and bounds recently. Workflow analysis of the microbiota with the molecular methods is shown in Figure 1. The development of the microbiota in the first year of life can affect long-term health, and can be influenced by perinatal factors, genetic, and diet, including foods and milk. The research that is conducted by Stearns et al. demonstrated that phylogenetically distinct groups of bacterial genera (in the order Lactobacillales vs Clostridiales) were seen in different abundances within each ethnic group (South Asian and Caucasian). Those bacterial taxa are good candidates to determine the effect of diet on microbiome, the effect of microbes on the host metabolism, and the stimulation of the bacteria to the host immune system in each group ethnicity. That research showed that differences in ethnicity can be mapped into the alfa-diversity, with an abundance of lactic acid bacteria, which were higher in South Asian ethnicity (Stearns et al., 2017).

South Asian ethnicity was verified to be originated from India, Pakistan, Sri Lanka, or Bangladesh, who settled in Canada for an average of 8 years, while Caucasian ethnicity settled in Canada for the rest of their life. Mothers of South Asian ethnicity are likely to be more vegetarians and diagnosed with gestational diabetes during pregnancy as well as receive antibiotics during delivery, whereas mothers of Caucasian ethnicity receive antibiotics during pregnancy. Neonates of South Asian ethnicity were born earlier, had a lower birth weight, and the increase in weight of the body during the first years of the first life were more substantial than neonates of Caucasian ethnicity (Stearns et al., 2017).

Some members of lactic acid bacteria (LAB), especially Bifidobacterium, Lactococcus, Streptococcus, and Enterococcus, are more abundant in South Asian neonates. LAB destroys carbohydrates that cannot be absorbed by the host into the acetate, lactate, or both, and are used as a source of energy for other groups of microbes. Besides that, the members of the phylum Actinobacteria are more abundance in South Asian neonates. Members of this phylum, such as Bifidobacteria, have been shown to dominate the gut microbiome of neonates. These bacteria are saccharolytic (which break down simple sugars) and have been shown to reduce their abundance in the microbiome of individuals with diet rich in fiber. Conversely, Caucasian neonates show an abundance of Firmicutes that is higher than that of Clostridiales, which shows an increase in response to diet withhigh animal protein and high fat. The fermentation products of acetate and lactate, known as indigestible fibers and oligosaccharides by members of the Clostridiales (Ruminococcus, Lachnospiraceae, and Oscillospira), include short chain fatty acids such as butyrate which are used by host cells as an energy source and an enhancement of barrier signal function (Stearns et al., 2017). Each species of lactic acid bacteria (LAB) has probiotic effect respectively (Sihotang and Fachrial, 2020). Other research shows that the gut microbiota contain strains of the genus/species of bacteria that have the potential as probiotics. Research conducted by Malik, et al. used meconium samples from Indonesian neonates that were analyzed using a molecular method. The research showed that the composition of Bacillus and Enterococcus faecalis was about $19.48 \%$ and 2,59\% of the total isolates of bacteria that were analyzed (Malik et al., 2020).

Population in an ethnicity refers to groups that include a mixture of dietary habits, cultural, language, religion, and ancestry. Many exposures that might lead to differences on the composition of the gut microbiota between ethnic groups include diet, health of the host, and the socioeconomic status (Byrd et al., 2020). Vegetarian diet is associated with the healthy and diverse gut microbiota, which are dominated by species that can metabolize carbohydrates that are not digested in the body. Meanwhile, a non-vegetarian diet (Western diet) is associated with a decrease in the abundance of Firmicutes and an increase in Bacteroides (Hasan and Yang, 2019). After ingestion of the Western diet, the microbiota ferments amino acids which results in the production of short chain fatty acids, as a source of energy, and harmful compounds. Vegetarian diets inhibit that process and encourage fermentation of carbohydrates as a primary function of gut microbiota (Clarke et al., 2019). A lowfiber Western diet can cause the weakening of the colon mucosal barrier so that it is resistant to pathogens and inflammation (Desai et al., 2016; Earle et al., 2015).

Effects of diet and lifestyle related to the population in an ethnic group appear during the 3 months after birth, even before given food companion. The significance of the influence will last up to 12 months in neonates from India and China. Based on the research that is conducted by $\mathrm{Xu}$ et al., microbiota of neonates from India is characterized by the higher abundance of Bifidobacterium and Lactobacillus, while neonates from China have a higher abundance of Bacteroides and Akkermansia (Xu et al., 2020). This finding provides a few details on the effect of specific and temporal factors, diet and lifestyle related to the population in an ethnicity in the development of the human gut microbiota. Table 1 shows the composition of the potential probiotics in the gut microbiota of neonates which were obtained from several ethnicities.

Some strains of probiotic bacteria can be observed from studies in Table 1, but most of the strains had not been tested for their potential probiotic activity. A good probiotic must meet the following criteria, such as not pathogenic, non-toxic, able to tolerate gastric 
acid, and can attach to the intestinal epithelium and produce antimicrobial compounds. Besides, probiotics have also to be able to survive quite a long time on the digestive tract to give a beneficial effect (Angelakis, 2017). Therefore, to be able to determine the activity of probiotic bacteria and its influence on the development of the health of the newborn, it is necessary to identify and further evaluate the potential probiotic bacterial isolates originating from neonatal meconium. The aim is that the potential probiotic bacteria that comes from the neonatal meconium can be clearly characterized by their activity and mechanism or the way they work in determining health outcomes for the development and growth of neonates in the future. Besides that, these potential probiotic bacteria can be developed as microbial therapeutic agents for the treatment of gastrointestinal impairments.

\section{CONCLUSION}

There are several potential probiotic strains in neonatal meconium microbiota such as Lactobacillus, Bifidobacterium, Enterococcus, Bacillus and Streptococcus. The composition of the gut microbiota or neonatal meconium microbiota is influenced by various factors including the differences in the mother's diet and lifestyle during pregnancy related to population and ethnicity. A vegetarian diet is associated with a diverse and healthy meconium microbiota that is predominantly bacteria with probiotic activity. Dietary differences affect the composition of probiotic bacteria in neonatal meconium. In the meconium microbiota of neonates from China and Japan, the composition of probiotic bacterial strains is more than that of ethnicities from other countries. The probiotic bacteria contained in the neonatal meconium microbiota serve as a mediator to increase the development of the neonatal innate and adaptive immune system responses, so that these effects can help prevent disease development in neonates.

The effect of these potential probiotic bacteria on the future health development of neonates can only be determined through large-scale prospective cohort studies involving different ethnicities with the help of more advanced technology. Molecular-based technologies, such as high-throughput sequencing, metagenomics, meta-transcriptomics, and metabolomics, can be used to understand the mechanisms of the effects of probiotics on gastrointestinal health and host immune function and to develop strategies to maximize the efficacy of probiotics in host health. Thus, potential probiotics in neonatal meconium microbiota could be developed as microbial therapeutic agents for the therapy of gastrointestinal impairments.

\section{ACKNOWLEDGMENT}

AM allowed writing part of her ongoing project and providing excellent scientific motivation and supervision to this manuscript. The division of Perinatology RSCM (CMH), especially Professor R. Rohsiswatmo and R. Amandito MD gave an excellent medical knowledge on neonate clinical cases. DO received PIMNAS research grant 2020 from the Ministry of Research and Technology of the Republic of Indonesia to study part of this topic.

\section{REFERENSI}

Alcon-giner, C. et al. (2017). Optimisation of 16S rRNA gut microbiota profiling of extremely low birth weight infants. BMC Genomics, 18(841), 1-15.

Angelakis, E. (2017). Weight gain by gut microbiota manipulation in productive animals. Microb. Pathogenesis 106, 162-170

Atarashi, K. et al. (2011). Induction of colonic regulatory $\mathrm{T}$ cells by indigenous Clostridium species. Science 331, 337-341.

Atya, A. K. Al et al. (2015) 'Probiotic potential of Enterococcus faecalis strains isolated from meconium', frontiers in Microbiology, 6(April).

Bain, C.C. et al. (2014). Constant replenishment from circulating monocytes maintains the macrophage pool in the intestine of adult mice. Nature Immunology 15, 929-937.

Bajaj, B.K., Claes, I.J., Lebeer, S. (2015). Functional mechanisms of probiotics. Journal of Microbiology, Biotechnology and Food Sciences, 4, 321-327.

Barnes, M.J. and Powrie, F. (2009). Regulatory T cells reinforce intestinal homeostasis. Immunity 31, 401-411

Belkaid, Y and Hand, T.W. (2014). Role of the microbiota in immunity and inflammation. Cell 157, 121-141.

Bene, K.P. et al. (2017). Lactobacillus reuteri surface mucus adhesins upregulate inflammatory responses through interactions with innate C-Type lectin receptors. Frontiers in Microbiology 8, 321

Bennett, C.L. et al. (2001). The immune dysregulation, polyendocrinopathy, enteropathy, X-linked syndrome (IPEX) is caused by mutations of FOXP3. Nature Genetics 27, 20-21. 
Bermudez-Brito, M., et al. (2012). Human intestinal dendritic cells decrease cytokine release against Salmonella infection in the presence of Lactobacillus paracasei upon TLR activation. PLoS One 7, e43197

Besser, J. et al. (2018) 'Next-generation sequencing technologies and their application to the study and control of bacterial infections', Clinical Microbiology and Infection, 24(4), 335-341.

Bimczok, D. et al. (2015). Human gastric epithelial cells contribute to gastric immune regulation by providing retinoic acid to dendritic cells. Mucosal Immunology, 8, 533-544

Birarra, M. K., Heye, T. B., and Shibeshi, W. (2017) 'Assessment of drug-related problems in pediatric ward of Zewditu Memorial Referral Hospital, Addis Ababa, Ethiopia', International Journal of Clinical Pharmacy, 39(5), 1039-1046.

Bleich, A. and Fox, J.G. (2015). The Mammalian Microbiome and its importance in laboratory animal research. ILAR Journal, 56, 153-158.

Boyle, R. J., Robins-browne, R. M., and Tang, M. L. K. (2018) 'Probiotic use in clinical practice: what are the risks ?', The American Journal of Clinical Nutrition, 83(April), 1256-1264.

Brittan, J.L. and Nobbs, A.H. (2015). Group B Streptococcus pili mediate adherence to salivary glycoproteins. Microbes and Infection, 17, 360-368.

Butel, M.-J., Waligora- Dupriet, A.-J., and WydauDematteis, S. (2018) 'The developing gut microbiota and its consequences for health', Journal of Developmental Origins of Health and Disease, (May).

Byrd, D. A. et al. (2020) 'Elucidating the role of the gastrointestinal microbiota in racial and ethnic health disparities', Genome Biology, 21(1), 192.

Campos, C. A. et al. (2006) 'Preliminary characterization of bacteriocins from Lactococcus lactis, Enterococcus faecium and Enterococcus mundtii strains isolated from turbot ( Psetta maxima ) q', Food Research International, 39, 356-364.

Caporaso, J. G. et al. (2012). Ultra-high-throughput microbial community analysis on the Illumina HiSeq and MiSeq platforms. The ISME Journal, 6(8), 1621-1624.

Clarke, G. et al. (2019) 'Gut reactions: Breaking down xenobiotic-microbiome interactions', Pharmacological Reviews, 71(2), 198-224.
Cleary, B. et al. (2016) 'Detection of low-abundance bacterial strains in metagenomic datasets by eigengenome partitioning', HHS Public Access, 33(10), 1053-1060.

Clemente, J.C., Ursell, L.K., Parfrey, L.W., and Knight, R. (2012). The impact of the gut microbiota on human health: an integrative view. Cell 148, 1258-1270.

Collado, M. C., Rautava, S., Aakko, J., and Isolauri, E. (2016). Human gut colonisation may be initiated in utero by distinct microbial communities in the placenta and amniotic fluid. Nature Publishing Group, (February), $1-13$.

de Agüero, M.G. et al. (2016). The maternal microbiota drives early postnatal innate immune development. Science 351, 1296-1302.

Desai, M.S. et al. (2016). A dietary fiber-deprived gut microbiota degrades the colonic mucus barrier and enhances pathogen susceptibility. Cell 167(5):13391353.

Diggikar, S. (2019) 'Neonatal Microbiome: A Complex, Invisible Organ and Its Evolving Role in Neonatal Illness and Beyond', Journal of Clinical Neonatology, 5-9.

Dwivedi, M., Kumar, P., Laddha, N.C., and Kemp, E.H. (2016). Induction of regulatory T cells: a role for probiotics and prebiotics to suppress autoimmunity. Autoimmunity Reviews, 15, 379-392.

ECDC/EMEA (2009). Joint Technical Report. The Bacterial Challenge: Time to React; European Centre for Disease Control: Stockholm, Sweden, September 2009.

Earle, K.A. et al. (2015). Quantitative imaging of gut microbiota spatial organization. Cell Host \& Microbe 18(4):478-488.

Fabricatore, A. N. et al. (2011) 'Intentional weight loss and changes in symptoms of depression : a systematic review and meta-analysis', International Journal of Obesity, 1-14.

Fakruddin, M., Hossain, N., and Ahmed, M. M. (2017) 'Antimicrobial and antioxidant activities of Saccharomyces cerevisiae IFST062013, a potential probiotic', BMC Complementary and Alternative Medicine, 1-11.

Fijan, S. (2016) 'Antimicrobial Effect of Probiotics against Common Pathogens', INTECH, (Probiotics and Prebiotics in Human Nutrition and Health).

Franzosa, E. A. et al. (2014) 'Relating the 
metatranscriptome and metagenome of the human gut', PNAS.

Gassler, N. (2017). Paneth cells in intestinal physiology and pathophysiology. World Journal of Gastrointestinal Pathophysiology, 8, 150.

Goodrich, J. K. et al. (2014) 'Conducting a Microbiome Study’, Cell, 158(2), 250-262.

Goodwin, S., Mcpherson, J. D. and Mccombie, W. R. (2016) 'Coming of age : ten years of next- generation sequencing technologies', Nature Publishing Group, 17(6), 333-351.

Gosalbes, M. J. et al. (2013) 'Meconium microbiota types dominated by lactic acid or enteric bacteria are differentially associated with maternal eczema and respiratory problems in infants', Clinical and Experimental Allergy, 43(2), 198-211.

Goto, Y. et al. (2014). Innate lymphoid cells regulate intestinal epithelial cell glycosylation. Science, 345, 1254009

Gratz, I.K. and Campbell, D.J. (2014). Organ-specific and memory treg cells: specificity, development, function, and maintenance. Front. Immuno. 5, 333.

Groer, M. W., Luciano, A. A., Dishaw, L. J., Ashmeade, T. L., Miller, E., and Gilbert, J. A. (2014). Development of the preterm infant gut microbiome : a research priority. Microbiome, 1-8.

Gupta, R. W. et al. (2013). Histamine-2 Receptor Blockers Alter the Fecal Microbiota in Premature Infants. JPGN, 56(4), 397-400.

Hasan, N. and Yang, H. (2019) 'Factors affecting the composition of the gut microbiota, and its modulation', PeerJ, 2019(8), 1-31.

Heilig, H.G., Zoetendal, E.G., Vaughan, E.E., Marteau, P., Akkermans, A.D., and de Vos, W.M. (2002). Molecular diversity of Lactobacillus spp. and other lactic acid bacteria in the human intestine as determined by specific amplification of $16 \mathrm{~S}$ ribosomal DNA. Applied and Environmental Microbiology, 68(1), 114-23.

Hojo, K., Nagaoka, S., Murata, S., Taketomo, N., Ohshima, T., and Maeda, N. (2007). Reduction of vitamin $\mathrm{K}$ concentration by salivary Bifidobacterium strains and their possible nutritional competition with Porphyromonas gingivalis. Journal of Applied Microbiology, 103, 1969-1974
Hooper, L.V., Littman, D.R., and Macpherson, A.J. (2012). Interactions between the microbiota and the immune system. Science, 336, 1268-1273

Hugenholtz, P. and Pace, N. R. (1996). Identifying microbial diversity in the natural environment : a molecular phylogenetic approach. Trends in Biotechnology, 14(96), 190-197.

Jostins, L. et al. (2012) 'Host-microbe interactions have shaped the genetic architecture of inflammatory bowel disease', Nature, 490(7422), 119-124.

Kankainen, M. et al. (2009). Comparative genomic analysis of Lactobacillus rhamnosus GG reveals pili containing a human-mucus binding protein. Proceedings of the National Academy of Sciences, USA 106, 171931719

Kau, A.L. et al. (2015). Functional characterization of IgA-targeted bacterial taxa from undernourished Malawian children that produce diet-dependent enteropathy. Science Translational Medicine, 7, 276 ra24.

Kaushik, J. K. et al. (2009) 'Functional and Probiotic Attributes of an Indigenous Isolate of Lactobacillus plantarum', PLoS One, 4(12).

Konieczna, P. et al. (2012). Bifidobacterium infantis 35624 administration induces Foxp3 T regulatory cells in human peripheral blood: potential role for myeloid and plasmacytoid dendritic cells. Gut 61, 354-366.

Kook, S. et al. (2019) 'Isolation and characterization of five novel probiotic strains from Korean infant and children faeces', PLoS One, 1-17.

Lécuyer, E. et al. (2014). Segmented filamentous bacterium uses secondary and tertiary lymphoid tissues to induce gut $\operatorname{IgA}$ and specific T helper 17 cell responses. Immunity, 40, 608-620.

Lebeer, S., Vanderleyden, J., and De Keersmaecker, S.C.J. (2010). Host interactions of probiotic bacterial surface molecules: comparison with commensals and pathogens. Nature Reviews Microbiology, 8, 171-184

Lee, Y.K. and Mazmanian, S.K. (2010). Has the microbiota played a critical role in the evolution of the adaptive immune system? Science, 330, 1768-1773.

Luo, C. et al. (2015) 'ConStrains identifies microbial strains in metagenomic datasets', Nature Biotechnology.

Mack, D.R., Michail, S., Wei, S., McDougall, L., and Hollingsworth, M.A. (1999). Probiotics inhibit 
enteropathogenic E. coli adherence in vitro by inducing intestinal mucin gene expression.American Journal of Physiology-Gastrointestinal and Liver, 276, G941-G950

Malik, A. et al. (2020). Clinical characteristics and clinical outcomes influence the cultivable-bacteria composition of the meconium of Indonesian neonates, Heliyon. [Manuscript].

Mantis, N.J., Rol, N., and Corthésy, B. (2011). Secretory IgA's complex roles in immunity and mucosal homeostasis in the gut. Mucosal Immunology, 4, 603611.

Marshall, K. (1977). Advances in Microbial Ecology. (R. M. Atlas, B. B. Jforgensen, J. H. Slater, \& K. M. Editor, Eds.) (Vol. 9). Kensington, Australia: MICROBIAL ECOLOGY Advances in Volume 9 Edited by K. C. Marshall University of New South Wales Kensington, New South Wales, Australia SPRINGER SCIENCE+ BUSINESS MEDIA, LLC.

Ma, T., Suzuki, Y., and Guan, L. L. (2018) 'Dissect the mode of action of probiotics in affecting host-microbial interactions and immunity in food producing animals', Veterinary Immunology and Immunopathology, 205, 35-48.

Marißen, J. et al. (2019) 'Efficacy of Bifidobacterium longum, B. infantis and Lactobacillus acidophilus probiotics to prevent gut dysbiosis in preterm infants of $28+0-32+6$ weeks of gestation : a randomised, placebo- trial : the PRIMAL Clinical Study protocol', BMJ Open, 9, 1-9.

Matamoros, S., Gras-leguen, C., Vacon, L., Potel, G., and Cochetiere, M. D. La. (2013). Development of intestinal microbiota in infants and its impact on health. Human Microbiome, 1-7.

Morais, J. et al. (2020) 'Extremely preterm neonates have more Lactobacillus in meconium than very preterm neonates-the in utero microbial colonization hypothesis', Gut Microbes, 12(1), 1-9.

Morgan, X. C. and Huttenhower, C. (2012) 'Chapter 12 : Human Microbiome Analysis’, PLoS Computational Biology, 8(12).

Mueller, N. T. et al. (2017) 'Delivery Mode and the Transition of Pioneering Gut-Microbiota Structure, Composition and Predicted Metabolic Function', Genes, 8(364).

Muñoz-Atienza, E. et al. (2013). Antimicrobial activity, antibiotic susceptibility and virulence factors of lactic acid bacteria of aquatic origin intended for use as probiotics in aquaculture. BMC Microbiology, 13, 15.

Nagpal, R. et al. (2016) 'Sensitive quantitative analysis of the meconium bacterial microbiota in healthy term infants born vaginally or by cesarean section', Frontiers in Microbiology, 7(DEC), 1-9.

O'Mahony, C. et al. (2008). Commensal-induced regulatory $\mathrm{T}$ cells mediate protection against pathogen-

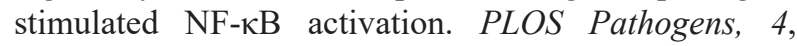
e1000112.

O'Sullivan, D.J. (1999). Methods for the analysis of the intestinal microflora. In: Tannock, G.W. (Ed.), Probiotics: A Critical 1997. Analysis of fecal populations of bifidobacteria and Review, Horizon Scientific Press, Norfolk, UK, 23-44.

Ohland, C.L. and MacNaughton, W.K. (2010). Probiotic bacteria and intestinal epithelial barrier function. American Journal of Physiology-Gastrointestinal and Liver, 298, G807-G819.

Okai, S. et al. (2016). High-affinity monoclonal IgA regulates gut microbiota and prevents colitis in mice. Nature Microbiology, 1, 16103.

Proft, T. and Baker, E.N. (2009). Pili in Gram-negative and Gram-positive bacteria - structure, assembly and their role in disease. Cellular and Molecular Life Sciences, 66, 613.

Round, J.L. and Mazmanian, S.K. (2010). Inducible Foxp3+ regulatory T-cell development by a commensal bacterium of the intestinal microbiota. Proceedings of the National Academy of Sciences USA 107, 12204-12209.

Russo, E., Taddei, A., Ringressi, M.N., Ricci, F., and Amedei, A. (2016). The interplay between the microbiome and the adaptive immune response in cancer development. Therapeutic Advances in Gastroenterology, 9, 594-605.

Rosser, E.C. et al. (2014). Regulatory B cells are induced by gut microbiota-driven interleukin- $1 \beta$ and interleukin-6 production. Nature Medicine, 20, 1334 1339.

Rintala, A. et al. (2017) 'Gut Microbiota Analysis Results Are Highly Dependent on the 16S rRNA Gene Target Region, Whereas the Impact of DNA Extraction Is Minor', Journal of Biomolecular Techniques, 28, 1930 .

Sakai, F. et al. (2014). Lactobacillus gasseri SBT2055 
induces TGF-b expression in dendritic cells and activates TLR2 signal to produce IgA in the small intestine. PLoS One 9, e105370.

Sarangi, A. N., Goel, A., and Aggarwal, R. (2019) 'Methods for Studying Gut Microbiota: A Primer for Physicians', Journal of Clinical and Experimental Hepatology, 9(1), 62-73.

Schlee, M., Harder, J., Köten, B., Stange, E.F., Wehkamp, J., and Fellermann, K. (2008). Probiotic lactobacilli and VSL\#3 induce enterocyte $\beta$-defensin 2. Clinical \& Experimental Immunology, 151, 528-535.

Schlee, M., Wehkamp, J., Altenhoefer, A., Oelschlaeger, T.A., Stange, E.F., and Fellermann, K. (2007). Induction of human $\beta$-defensin 2 by the probiotic Escherichia coli Nissle 1917 is mediated throuah flagellin. Infection and Immunity, 75, 2399-2407.

Shaw, M.H., Kamada, N., Kim, Y.G., and Núñez, G. (2012). Microbiota-induced IL-1 $\beta$, but not IL-6, is critical for the development of steady-state TH17 cells in the intestine. Journal of Experimental Medicine, 209, $251-258$.

Shokryazdan, P. et al. (2014) 'Probiotic Potential of Lactobacillus Strains with Antimicrobial Activity against Some Human Pathogenic Strains'.

Sihotang, S. and Fachrial, E. (2020) 'Isolasi, identifikasi dan karakterisasi bakteri probiotik dari mekonium isolation, identification and characterization of probiotic bacteria from meconium', Jurnal Kedokteran STM (Sains dan Teknologi Medik), 3(2), 82-90.

Smolinska, S., Groeger, D., and O'Mahony, L. (2017). Biology of the microbiome 1: interactions with the host immune response. Gastroenterology Clinics of North America, 46, 19-35

Stearns, J. C. et al. (2017) 'Ethnic and diet-related differences in the healthy infant microbiome', Genome Medicine, 9(23), 1-12.

Tannock, G.W. (1999). Identification of lactobacilli and bifidobacteria. In: Probiotics: A Critical Review, Horizon Scientific Environ. Microbiology, 64, 4816-4819. Press, Norfolk, UK, 45-56
Tsai, Y.T., Cheng, P.C., and Pan, T.M. (2012). The immunomodulatory effects of lactic acid bacteria for improving immune functions and benefits. Applied Microbiology and Biotechnology, 96, 853-862.

Turroni, F. et al. (2014). Expression of sortase-dependent pili of Bifidobacterium bifidum PRL2010 in response to environmental gut conditions. FEMS Microbiology Letters, 357, 23-33.

Varankovich, N.V., Nickerson, M.T., and Korber, D.R. (2015). Probiotic-based strategies for therapeutic and prophylactic use against multiple gastrointestinal diseases. Frontiers in Microbiology, 6, 685.

Viladomiu, M. et al. (2017). IgA-coated E. coli enriched in Crohn's disease spondyloarthritis promote TH17- dependent inflammation. Science Translational Medicine, 9, eaaf9655.

Weaver, C.T., Harrington, L.E., Mangan, P.R., Gavrieli, M., and Murphy, K.M. (2006). Th17: an effector CD4 $\mathrm{T}$ cell lineage with regulatory $\mathrm{T}$ cell ties. Immunity, 24, 677-688.

White, J. R., Nagarajan, N., and Pop, M. (2009) 'Statistical Methods for Detecting Differentially Abundant Features in Clinical Metagenomic Samples', PLoS Computational Biology, 5(4).

$\mathrm{Xu}$, J. et al. (2020) 'Ethnic diversity in infant gut microbiota is apparent before the introduction of complementary diets', Gut Microbes, 1-12.

Yan, F., Cao, H., Cover, T.L., Whitehead, R., Washington, M.K., and Polk, D.B. (2007). Soluble proteins produced by probiotic bacteria regulate intestinal epithelial cell survival and growth. Gastroenterology,132, 562-575.

Zavisic, G. et al. (2019) 'Probiotic potential of Lactobacillus fermentum G-4 originated from newborns meconium', Journal of the Serbian Chemical Society, 2020(February).

Zhao, Q. and Elson, C.O. (2018). Adaptive immune education by gut microbiota antigens. Immunology, 154, 28-37. 